\title{
Feasibility of LoRa Implementation for Remote Weather Monitoring System through Field Measurement and Case Study Analysis
}

\author{
N.H. Abd Rahman 1,2,*, Y. Yamada', M.H. Husni' ${ }^{2}$, N.H. Abdul Aziz ${ }^{2}$ \\ ${ }^{1}$ Malaysia-Japan International Institute of Technology, \\ Universiti Teknologi Malaysia, Jalan Sultan Yahya Petra, Kuala Lumpur, 54100, MALAYSIA \\ ${ }^{2}$ Faculty of Electrical Engineering, \\ Universiti Teknoogi MARA, Shah Alam, Selangor, 40450, MALAYSIA \\ *Corresponding Author
}

DOI: https://doi.org/10.30880/ijie.2018.10.07.027

Received 31 July 2018; Accepted 25 November 2018; Available online 30 November 2018

\begin{abstract}
This paper discusses the feasibility of Long Range Wide-area network (LoRa-WAN) implementation for weather data collection and transmission in remote locations, specifically in east Malaysia. Currently, Malaysia Meteorological Department (MetMalaysia) has installed more than 100 weather stations around Malaysia, and out of this number, there are many stations, mostly in east Malaysia that are dependent on Very Small Aperture Terminal (VSAT) services for data transmission due to unavailability of mobile coverage. Due to significant cost of VSAT subscription, LoRa communication is proposed as an alternative solution due to its low power, low-cost, and long range characteristics. In this paper, the study has been performed through two stages; first, technical performance of LoRa network was validated through field measurement in urban areas to determine the actual LoRa characteristics in real condition. In the measurement, a LoRa module operating at $433 \mathrm{MHz}$ was used to demonstrate a single channel LoRa gateway that acted as a bridge to communicate between LoRa node to IP network. Parameters such as received power spectrum and free space path loss were recorded for analysis. Second, based on the measured data, extrapolation of maximum feasible distance of LoRa signal propagation was done to analyze the viability of implementing LoRa in various locations of existing weather stations in Sabah and Sarawak. Two case studies are presented in this paper, with the aim to evaluate the suitable location of LoRa gateway to establish communication with the existing weather stations when LoRa network is used. Detailed link budget analysis was performed for each scenario. Based on the comparison between theoretical and measurement, the power transmitted from LoRa node shows inconsistent readings throughout the day, suspected due to the changes in spread factor that has been automatically set by the module. Nevertheless, taking into account this factor, it was found that the transmission range was adequate for some remote locations. Meanwhile, for some locations that are separated too far from each other, higher power is needed to allow consistent communication.
\end{abstract}

Keywords: LoRa, link budget, IoT, UHF, propagation

\section{Introduction}

Internet of Things (IoT) has widened many areas of research such as cloud computing, robotics, big data and semantic $[1,2]$. Over the years, IoT application has cleared a path for fast and real-time analysis of weather data which is useful for weather forecasts. In Malaysia, the Malaysia Meteorological Department (MetMalaysia) has installed more than 
hundred (100) Automatic Weather Station (AWS) at many remote locations as shown in Figure 1(a) to analyze weather data gathered from various sensors such as thermometer, anemometer, wind vane, hygrometer and gauge [3]. The information obtained at each station will be transmitted frequently to home office through either satellite or broadband network, depending on the locations. The utilization of broadband technology provided by telecommunication companies (Telco) can suit most AWS in Malaysia particularly in the urban region, which allows forecasters to observe the genuine situation in specific locations. Here, GSM/GPRS empowered SIMs were installed in the AWS terminal to wirelessly transfer information of data logger to the cloud. For a remote site that has no coverage of Telco's base station, satellite transmission through VSAT has been employed. Data communication through VSAT system offers a reliable solution however, the subscription cost is very high, and to cover around 25 remote locations around Malaysia that currently have no broadband access is definitely costly. Figure 1(b) illustrates the location of AWS in remote locations, where 7 stations are in Peninsular Malaysia (West Malaysia) and 18 stations are located in Borneo region (East Malaysia).

a

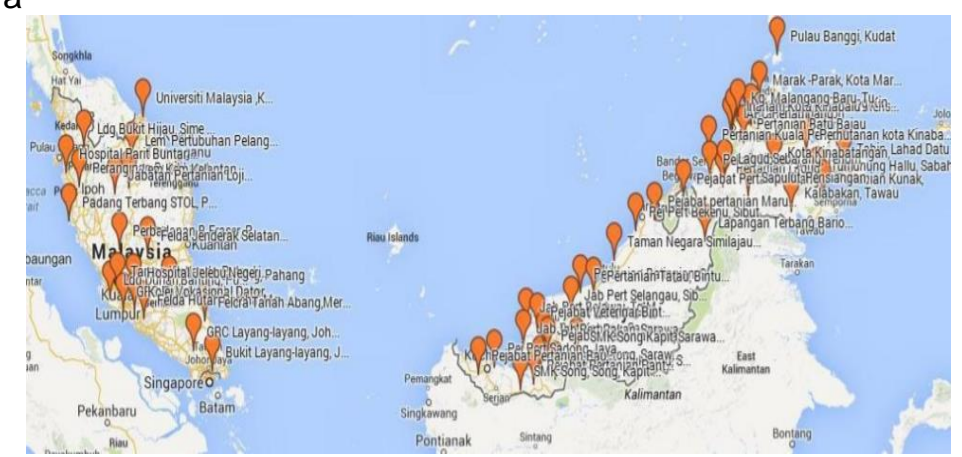

b

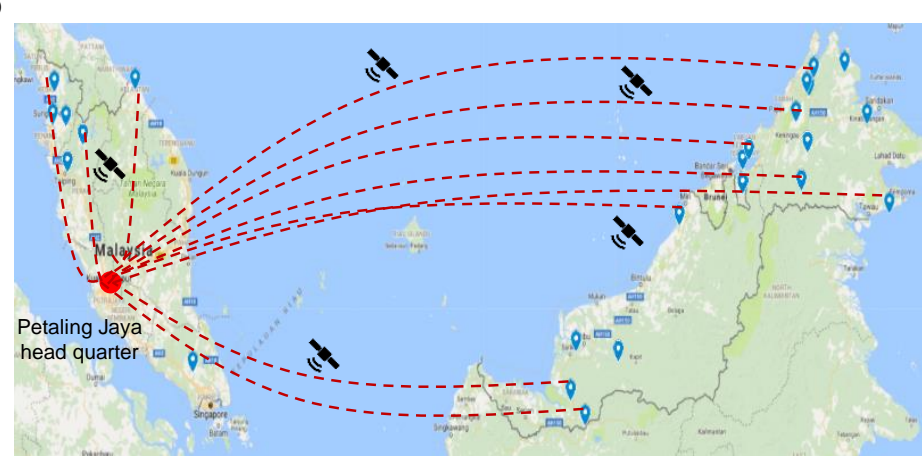

Fig. 1 - (a) Locations of Automatic Weather Station (AWS) in Malaysia [3]; (b) Locations of AWS that utilize VSAT service for weather data transmission to head quarter in Petaling Jaya.

Long Range Wide Area Network (LoRaWAN) is a wireless technology that operates below $1 \mathrm{GHz}$, which can be used to transmit signal at low power level, hence can lower the power consumption and broaden battery lifetime [4,5]. Many research works on the reliability of LoRa for long distance communication have been conducted recently such as in [6-8]. LoRa utilizes asynchronous communication to keep up so the hub will only 'wake up' when they need to send information and will go back into power saving 'sleep mode' straightforwardly or once the transmitted information has been recognized. Depending on the earth condition and the presence of obstacles, the LoRa module used in this paper is stated to be able to cover up to $15 \mathrm{~km}$ radial distance in rural zones and more than $5 \mathrm{~km}$ in urban conditions. To verify the information given and to relate with current situation in remote areas, the measurement of received signal strength indicator (RSSI) was performed in this paper and the free space path loss (FSPL) calculation was analyzed. Through the measured RSSI data and detailed link budget analysis, the feasibility of LoRa communication as a solution for weather data transmission in remote areas was investigated and studied. The applicability of this technology in real situation was assessed based on the current locations of remote weather stations in Sabah and Sarawak.

\section{Development of LoRa-WAN Model}

The section is divided into three (3) segments; system architecture, hardware design and software configuration. System architecture describes the LoRa network in general, based on the communication between LoRa node(s) with sensors, and transmission of data from LoRa nodes to LoRa gateway. In this project, for the demonstration purpose, only one LoRa node was utilized and two sensors were used. In the hardware segment, all tools and equipment related to this project is described. In this project, LG01 with LoRa Shield manufactured by Dragino was used. Arduino Uno, a 
microcontroller board based on the ATmega328P was used to integrate all sensors. All debugging and compiling processes were done in Arduino IDE tool. For instant visualization of data, an online platform called ThinkSpeak is used, however, this tool can only display sensor data when connection is established. Meanwhile, in order to obtain radio frequency parameters such as received power, a spectrum analyzer with an omni-directional dipole antenna is utilized.

\subsection{System Architecture}

Fig. 2 shows the basic block diagram of the system. To demonstrate the data transmission capability, Arduino module integrated with various weather sensors is incorporated together with a LoRa Shield that acts as a node to transmit all collected data to LoRa gateway, LG01. All data will then be uploaded wirelessly to the server, depending on the connectivity between LoRa gateway to the cloud.

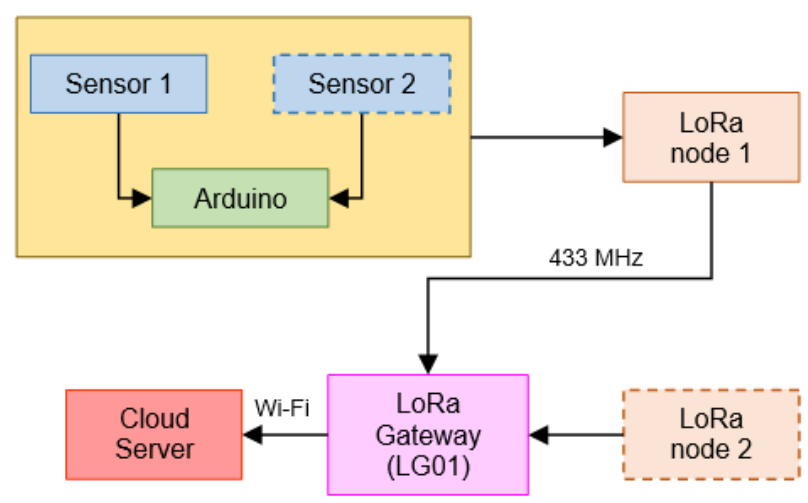

Fig. 2 - Block diagram of system design.

Table 1 shows the specification of LoRa module used in this project [9].

Table 1 - LoRa module and hardware specification [9].

\begin{tabular}{lc}
\hline Description & Detailed specification \\
\hline Hardware system & Linux: 400Mhz ar9331 Processor, 64MB RAM, 16MB Flash \\
& Microcontroller unit (MCU): ATMega328P, Flash 32KB, \\
SRAM 2KB, EEPROM 1KB & Power Input: 9-24v DC, 2 x RJ45 Ports, USB 2.0 Host port \\
Interface & Freq Range: $410-528 \mathrm{Mhz}$ \\
LoRa module & RF output: $+20 \mathrm{dBm}-100 \mathrm{~mW}$ constant \\
Power amplifier: $+14 \mathrm{dBm}$ high-efficiency \\
Programmable bit rate up to $300 \mathrm{kbps}$ \\
High sensitivity: down to -148 dBm \\
Low RX current of $10.3 \mathrm{~mA}$ \\
FSK, GFSK, MSK, GMSK, LoRaTM and OOK
\end{tabular}

\subsection{Hardware Design}

LG01 is an open source single channel LoRa gateway acting as a bridge of connection from one or more node(s) to IP network that is based on WiFi, Ethernet, $3 \mathrm{G}$ or $4 \mathrm{G}$ cellular. The overview of LG01 operation is illustrated in Fig. 3. In this project, a low-frequency LoRa variant operating at $433 \mathrm{MHz}$ was used. In this proof-of-concept module, two types of sensors were integrated with the LoRa node. The DHT11 temperature and humidity sensor were used to provide calibrated digital signal output. An 8-bit microcontroller was used to control and monitor both sensors. Program uploading and debugging process were done via Arduino IDE. 


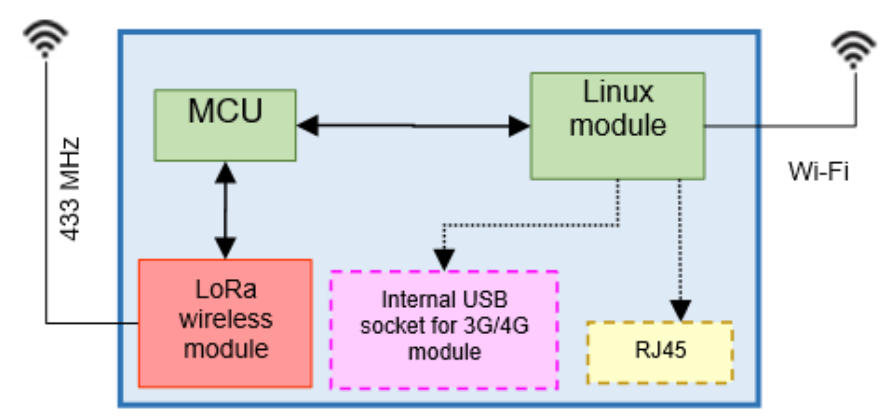

Fig. 3 - Block diagram of LoRa gateway, LG01.

This LoRa module comes with a data-processing tool called ThingSpeak that allows accumulation and analysis of data collected in cloud with third-party software like MATLAB [8]. For this project, data received at LoRa gateway were observed through ThingSpeak and real-time performance of the LoRa frequency spectrum was measured by using Keysight's spectrum analyzer. Fig. 4 shows the test setup for measuring spectrum performance of the LoRa system such as power received, power transmitted and frequency of operation. By using the measured data, maximum radial range of the system will be determined through link budget calculation. The free space path loss (FSPL) was calculated using the following formula [2], where $R$ is the distance between transmitter (LoRa node) and receiver (LoRa gateway), $f$ is the frequency of operation, $c$ is the speed of light, $G_{T X}$ is the transmit antenna gain in $\mathrm{dB}$ and $G_{R X}$ is the receive antenna gain in $\mathrm{dB}$ :

$$
F S P L[d B]=20 \log _{10} R+20 \log _{10} f+20 \log _{10}\left(\frac{4 \pi}{c}\right)-G_{T X}-G_{R X}
$$

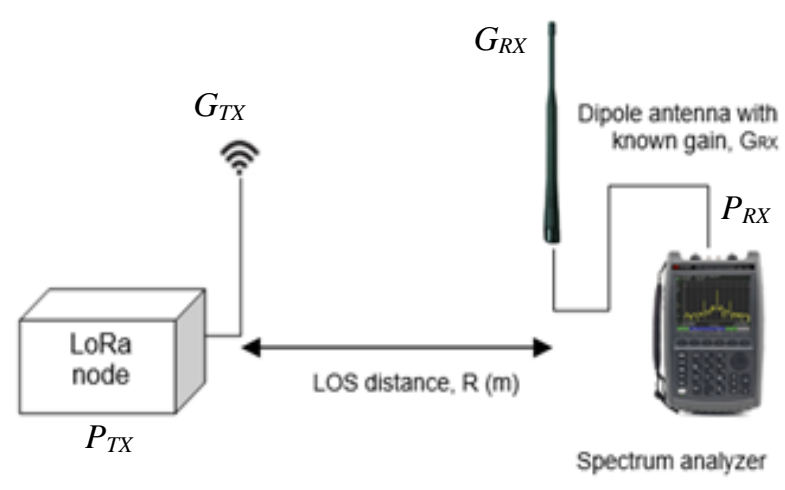

(a)

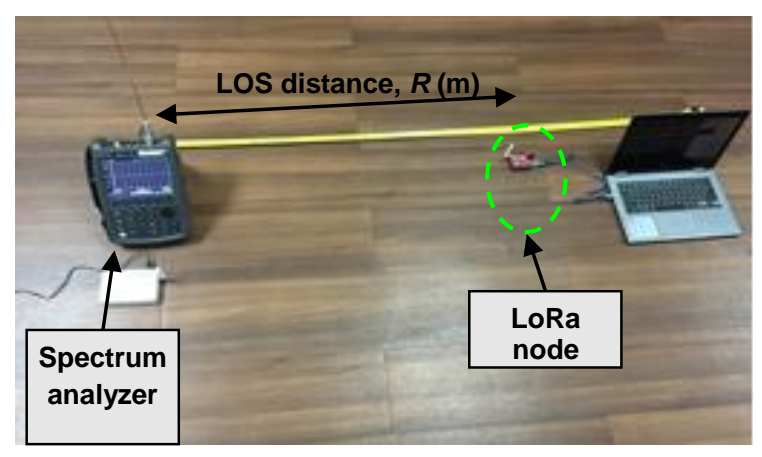

(b)

Fig. 4 - Measurement setup for spectrum performance and link budget.

\subsection{Software Configuration}

On the first boot, the LG01 gateway was connected to a designated WiFi network and once connected, user can get access to the default IP address for the gateway. A web-based tool called PuTTy was used to configure and alter gateway's internet access from a given IP address. Here, a specific IP address, e.g. Dragino was entered. This step was important during troubleshooting to make sure that the gateway setting was not the reason if anything fails. It can also be used to monitor gateway's activity such as its network internet connection, memory, and type of connection used [9]. WiFi Client Mode was chosen in this project because for demonstration purposes, an active internet connection from any kind of router is sufficient. For wireless connection, DHCP option was used instead of static IP to allow data transmission to the cloud server. 


\section{Analysis of Issues and Results}

This section is divided into three (3) segments; communication between LoRa node and gateway, results on application server, and radio frequency analysis.

\subsection{Communication between LoRa Node and Gateway}

The main task was to ensure that the prototype can work as a system including Arduino board and sensors. There were some problems and issues encountered, for example:

i. No communication between LoRa node and LoRa gateway

ii. Data collected by sensors could not be transmitted to cloud server

Series of tests and system troubleshooting have been done to identify the problem. Table 2 shows the steps that have been conducted in sequence:

Table 2 - Troubleshooting sequence.

\begin{tabular}{lcc}
\hline No. & Actions & Indicator \\
\hline 1 & $\begin{array}{c}\text { To ensure all sensors worked } \\
\text { properly (standalone) }\end{array}$ & $\sqrt{ }$ \\
2 & $\begin{array}{l}\text { Use simpler codes to test the } \\
\text { communication between node } \\
\text { and gateway }\end{array}$ & $\sqrt{ }$ \\
3 & Check and validate the codes & $\sqrt{ }$ \\
4 & Check gateway setting (e.g. & $\times$ \\
& IP address, mode etc) & \\
\hline
\end{tabular}

It was found that the gateway was not connected to the internet so no data can be sent to the cloud. There was an error when choosing the gateway mode of obtaining its IP address. Static IP shall be selected when the connection was wired and 'DHCP' shall be chosen when it was connected wirelessly.

\subsection{Results on Application Server}

Data obtained from both sensors were sent to the cloud (ThingSpeak) via LoRa gateway. LoRa gateway acted as a medium for the collected data from LoRa node (sensors and Arduino) to be displayed in the cloud. The sensor that was used for the test was DHT11 to measure the temperature and humidity of the surrounding. Since the objective of this project is to promote LoRa gateway usage in weather monitoring system, DHT11 was chosen as an example of the actual sensor that will be used in real application. More sensors can be added in future to further maximize LoRa gateway and Arduino capability

Fig. 5 shows the received sensor data, plotted against time. As demonstrated, the number of dots represent the obtained data which are very consistent, however as shown by the dotted circles, there are times when no data is being displayed or received, and this happens for a short duration. The gap can be from one second up to 30 minutes which indicates that the data may sometimes be lost during communication.

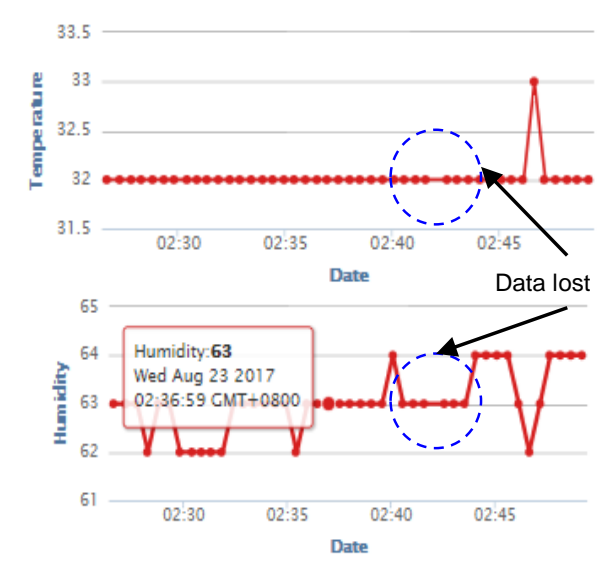

Fig. 5 - Real time data of temperature and humidity.

There are a number of factors causing this scenario to happen. Firstly, it could be because of the internet connectivity. It is possible that internet connection by some internet service providers suddenly suffer intermittence or slower 
connection speed. There could also be signal interference which requires the device to continuously resend messages to overcome signal issues, thus create delays.

Another factor could be due to the LoRa itself. One of its features is its capability to turn into deep sleep mode to save power when there are no data to be sent to the cloud. As a result, no data will be received thus create hiatus for some period of time. However, this feature could be modified in the coding to avoid this problem to occur. Although weather data is not considered as 'critical', the stability of LoRa to replace VSAT for weather data transmission by Malaysia Meteorological Department (MetMalaysia) shall be assessed further and be improved because definitely a reliable solution is preferred.

\subsection{Radio Frequency and Link Budget Analysis}

Radio frequency and propagation measurement were required to verify LoRa's capability and viability to work in the forests or rural areas for VSAT replacement. Link budget is a simplified design tool that can be used to provide theoretical assessment of the feasibility of this system. However, this tool does not account for real-world variables that may affect propagation performance, such as non-line of sight (LOS) factors, atmospheric attenuation and many more. Nevertheless, in this paper, in order to determine the maximum distance for signal propagation, minimum fade margin (FM) of between 20 to $30 \mathrm{dBm}$ shall be obtained. During measurement, the distance between LoRa node(s) and gateway was varied and the received power was measured and recorded to calculated the link budget and fade margin. If the fade margin is less than $10 \mathrm{dBm}$ for certain distance, it indicates that the propagation has reached its maximum distance. The formula for fade margin (FM) is shown as in equation (2):

$$
\text { Fade margin }=P_{R X}-R X \text { sensitivity }
$$

Keysight's Fieldfox spectrum analyzer with sensitivity of $-110 \mathrm{dBm}$ was used as the main equipment to measure LoRa's received power with respect to various distance. The theoretical reason behind the experiment and taking into account Friis transmission equation, as distance increases the power received decreases. Therefore, the results can be used to measure the maximum distance of the transmission signal to get an estimation of LoRa's capability to transmit a signal over a long distance.

Measurement for the best condition of signal transmission was done by using the wired-communication from LoRa node to spectrum analyzer. As shown in Fig. 6, the power received or known as Received Signal Strength Indicator (RSSI) obtained is $7.304 \mathrm{dBm}$. However, in the ideal condition the power transmission according to the datasheet was supposed to be $14 \mathrm{dBm}$. Even with cable, there were losses that occurred thus deteriorates the value. This factor has to be taken into consideration in determining the feasibility of the device to replace VSAT when there may be an obstruction and other factors that can cause signal loss. To further examine LoRa signal, the same test was held wirelessly to check the value of power received in actual condition. This time, instead of using a cable, an antenna with known gain, $\mathrm{GRX}=2.15 \mathrm{dBi}$ was connected to spectrum analyzer and the distance was varied from 1 meter to 10 meters. The measured RSSI values and the calculated FSPL are displayed in Fig. 7. Based on the graph, it shows that as the distance increases, the RSSI decreases which is true. However, there are a few times when the power received are significantly low as observed at 2 meters, 4 meters and 5 meters. This result shows that there are some inconsistencies in signal propagation through free space. The measured values of $P_{R X}$ is compared with the theoretical data when the transmit power $P_{T X}$ is assumed to be $7.304 \mathrm{dBm}$ according to the obtained result when cable is connected. The comparison is shown in Fig. 8 . Here, significant difference between calculated and measured data for $P_{R X}$ is shown. Fig. 9 shows the comparison of FSPL between theoretical and measurement by varying the distance $R$. Here, both values of FSPL seem consistent and no significant deterioration observed.

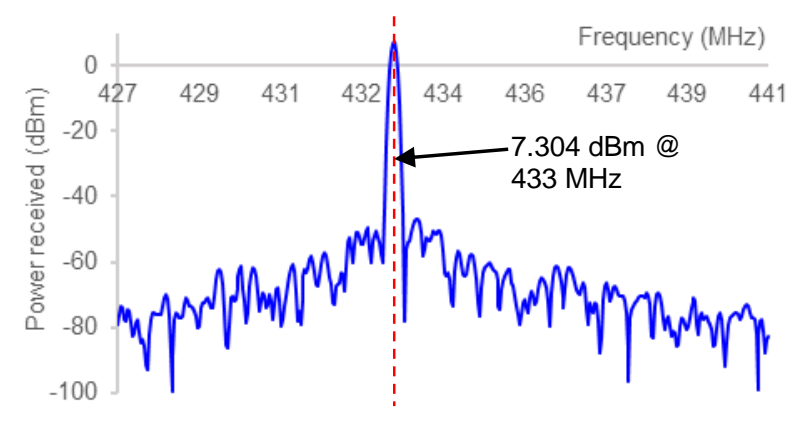

Fig. 6 - Power Received with Cable. 


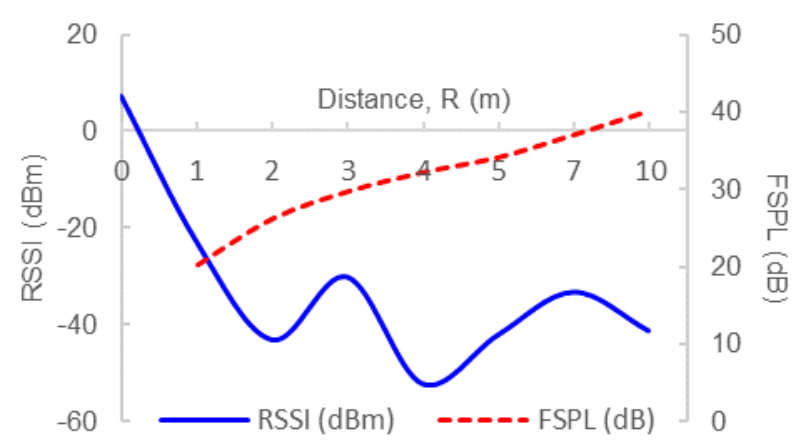

Fig. 7 - RSSI and FSPL for various distance.

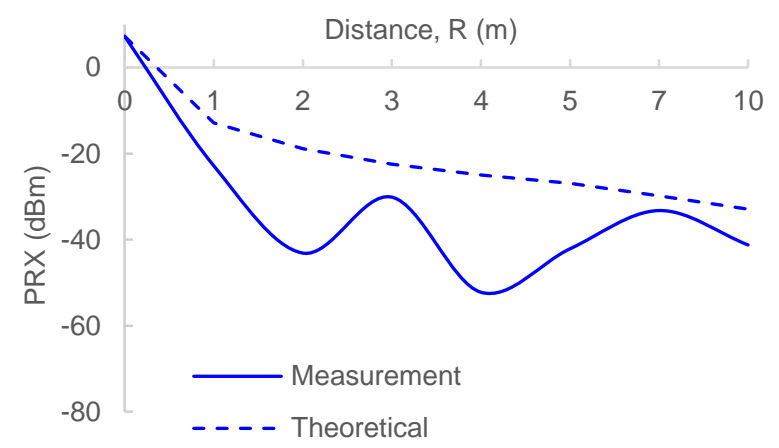

Fig. 8 - Comparison between measured and theoretical received power $\left(P_{R X}\right)$ for various distance.

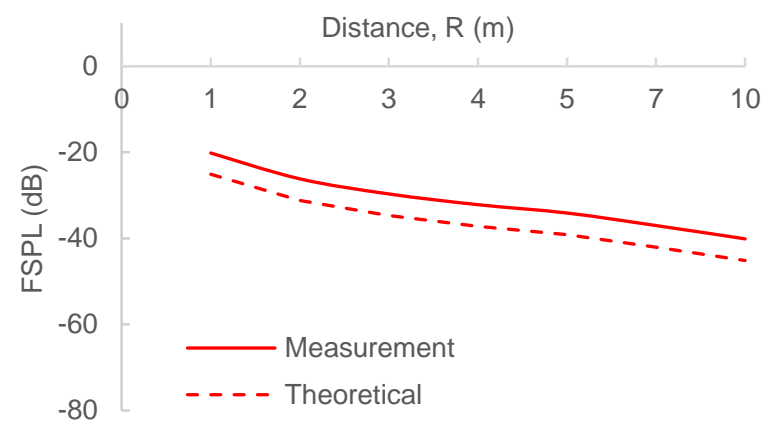

Fig. 9 - Comparison between measured and theoretical FSPL for various distance.

There are multiple reasons that may contribute to this phenomenon, it could be due to Doppler effect, multipath fading, scattering, interferences from other radio systems that operate on $433 \mathrm{MHz}$ band or simply an obstruction from its line of sight when taking readings. Nonetheless, necessary measures have been taken to minimize possible errors that might cause the last factor to occur and clear line of sight was ensured. For link budget analysis, it was assumed that LoRa node transmitted the same amount of power throughout the experiment $\left(P_{T X}=7.3 \mathrm{dBm}\right)$ and the same value was used to measure the maximum distance. With that assumption, predictions can be made using the Friis Transmission Equation, which is [2]:

$$
P_{R X}=\frac{P_{T X} G_{T X} G_{R X} \lambda^{2}}{(4 \pi R)^{2}}
$$

The formula was manipulated to find the distance of signal transmission, $R$. The comparison of $P_{T X}=7.3 \mathrm{dBm}$ and 14 $\mathrm{dBm}$ were made to further analyze the potential of LoRa. Table 3 shows the comparison of maximum distance when power transmit, $P_{T X}$ is $7.3 \mathrm{dBm}$ and $14 \mathrm{dBm}$ respectively. This values are calculated based on the actual transmit power $P_{T X}$ of $7.3 \mathrm{dBm}, P_{R X}$ of $-100 \mathrm{dBm}$ to ensure fade margin (FM) of $10 \mathrm{~dB}$ minimum, and antenna gain of $2.5 \mathrm{dBi}$ for each $G_{R X}$ and $G_{T X}$. There are many methods to improve the propagation distance such as by increasing the gain of both transmitting and receiving antennas. As shown in equation (3), when the antenna gains $\left(G_{R X}\right.$ and $\left.G_{T X}\right)$ are increased, the received 
power, $P_{R X}$ will increase thus fade margin will be higher and larger distance can be covered. However, any modifications made shall take into account the Effective Isotropic Radiated Power (EIRP) limit set by Federal Communications Commission (FCC) for this unlicensed spectrum band. For frequency range of 433 to $435 \mathrm{MHz}$, the maximum EIRP is $100 \mathrm{~mW}$. Other than antenna gain, deterioration in received power may possibly due to the multipath interference or nonline-of-sight NLOS) factor, mainly because there are many obstructions or obstacles nearby. Due to the low frequency used in the system, even small changes in half or one wavelength could significantly affect the received power level. To make the LoRa network more efficient, another improvement would be to add more LoRa gateway to the path and configure it as a repeater to send data to the final gateway that has internet connectivity.

Table 3 - Comparison of maximum distance.

\begin{tabular}{lc}
\hline $\begin{array}{l}\text { Power Transmit, } \boldsymbol{P}_{T X} \\
(\mathbf{d B m})\end{array}$ & Distance, $\boldsymbol{R}(\mathbf{k m})$ \\
\hline $7.3 \mathrm{dBm}$ & $23 \mathrm{~km}$ \\
$14 \mathrm{dBm}$ & $49.5 \mathrm{~km}$ \\
\hline
\end{tabular}

\section{Case Study}

Case study analysis was performed to assess the viability of replacing VSAT with LoRa. Table 4 shows the existing locations of VSATs in Sabah and Sarawak that have been installed by MetMalaysia to transmit their weather data from remote weather stations to the headquarter in Petaling Jaya. VSATs have been used in these locations due to the unavailability of telecommunication network (base stations) in those areas.

Table 4 - Existing VSAT locations in Sabah and Sarawak.

\begin{tabular}{lccc}
\hline No. & Names of stations & \multicolumn{2}{c}{ Exact positions } \\
\cline { 3 - 4 } & & Latitude & Longitude \\
\hline 1 & Lubok Antu & 1.04015 & 111.83864 \\
2 & Betong & 1.41125 & 111.5306 \\
3 & Bekenu Sibuti & 4.05658 & 113.844 \\
4 & SMK Song & 2.00495 & 112.555 \\
5 & Sundar & 4.89136 & 115.204 \\
6 & Bintagor & 2.1613 & 111.64447 \\
7 & Serinsim & 6.29319 & 116.70702 \\
8 & Mensilau & 6.00518 & 116.61034 \\
9 & Batu Bajau & 5.61896 & 116.32735 \\
10 & Kuala Penyu & 5.60539 & 115.59198 \\
11 & K. Kinabatangan & 5.58158 & 117.85341 \\
12 & Laban Rata & 6.06012 & 116.56608 \\
13 & Simpang Paitan & 6.37643 & 117.37028 \\
14 & Salong & 4.58085 & 116.45486 \\
\hline
\end{tabular}

As calculated before, the maximum distance of LoRa range is up to $23 \mathrm{~km}$ with $7.3 \mathrm{dBm}$ power transmitted. With that information, the locations of VSAT were investigated in this project to observe LoRa's ability to cover the area needed. However, LoRa gateway can only function with the availability of internet connectivity to transmit data to the cloud. It could be a deciding factor when placing LoRa at a remote location because there will be no network available hence making it less ideal to replace VSAT.

\subsection{Case 1 (Mount Kinabalu)}

One of the ideal locations to install LoRa gateway is at the Mount Kinabalu Golf Club. This is due to the fact that it is the tourism center of Sabah, thus most likely the internet connection is also available. Besides, based on the calculated $23 \mathrm{~km}$ radius, it can cover two existing VSAT stations; Laban Rata and Mensilau which are not far away (only $5 \mathrm{~km}$ and $3 \mathrm{~km}$ away respectively). Fig. 10 shows the distance of the existing VSAT locations from the possible of LoRa gateway location retrieved from Google Maps. 


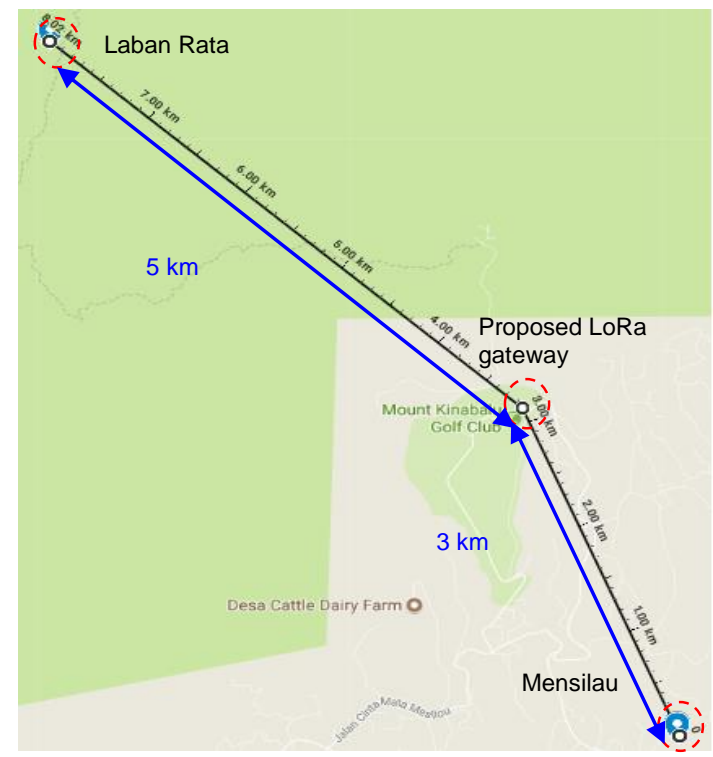

Fig. 10-Distance of existing VSAT locations (Mensilau and Laban Rata) to proposed LoRa gateway location.

\subsection{Case 2 (Sundar)}

Another proposed location is SMK Sundar. Since the current VSAT is technically located in the city of Sundar, LoRa can be placed anywhere in the city. Fig. 11 shows the distance of SMK Sundar to current VSAT location. It was chosen because it would provide a much better elevation thus making it easier to receive data that is transmitted from the node located only 800 meters away. However, it can only cover one current VSAT location. There are other places that will be suitable to place LoRa gateway that would promise internet connectivity. However, like mentioned previously, it would be able to cover only one VSAT per location, hence not utilizing LoRa to its full potential unless more AWS is installed at new locations. One of the best features of LoRa is its capability to connect with hundreds of different nodes at the same time where a number of existing VSATs (nodes) can just use one gateway to transmit data to the cloud. However, its implementation is limited to the maximum radial distance that it can cover. Nevertheless, the implementation of LoRa would still be much cheaper than VSAT even though it covers only one VSAT per location.

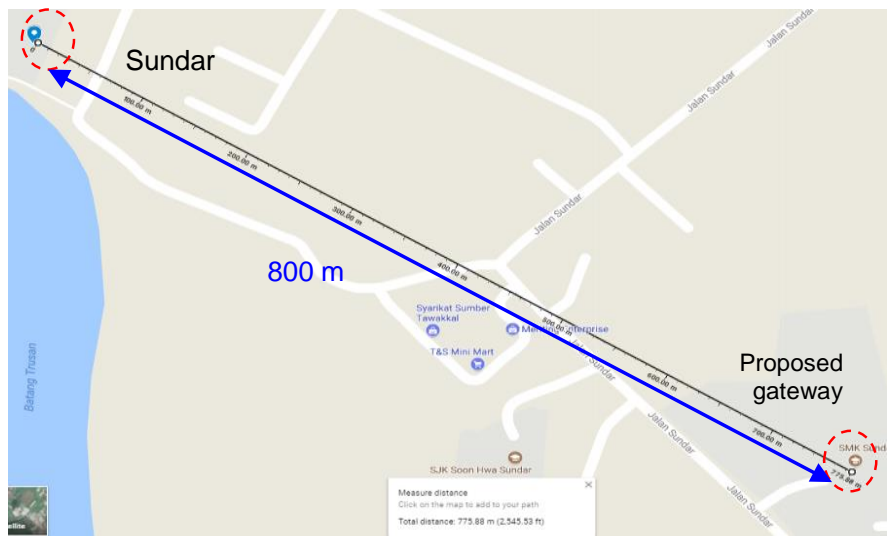

Fig. 11- Distance of proposed gateway location to existing VSAT station (Sundar).

\section{Conclusion and Recommendation}

To conclude, LoRa is obviously a great technology to be used as an alternative for communication as it offers a cheap and efficient way to transmit data to the cloud. The presented results have highlighted the important features and limitations of LoRa technology. More research needs to be done in the future to help decide its feasibility. For example, in this project, its spread factor (SF) was set by default, where LoRa would decide what type of SF from $7-12$ that would be ideal to use in that situation. In the future, SF could be optimized, and analysis of its difference between those values could be investigated. In theory, $\mathrm{SF}=12$ would give a longer range but fewer packets of data will be received, while SF $=7$ would be efficient to transmit data all the time but limited to certain distance. Besides that, measurements of a different mode of bandwidths, modulation techniques, and even different environments could be tested. One of the findings of the project was the value of power transmitted obtained are not the same as the data sheet. It's proven that this is vital as it 
can greatly improve the maximum distance of data transmission. Thus, improvement in hardware design can be done to improve its performance. One of the ways to do it is by replacing the antenna of the gateway and node. This could improve the characteristics of the signals transmitted, gain of the antenna, thus improve the results of the findings.

\section{Acknowledgement}

Authors would like to thank Universiti Teknologi Malaysia for supporting this project under the Professional Development Research University Grant with grant no. PY/2017/02232. Special thanks to Malaysia Meteorology Department (MetMalaysia) for providing data on the existing VSAT locations for weather monitoring around Malaysia.

\section{References}

[1] Kocovic, P., Behringer, R., Ramachandran, M., \& Mihajlovic, R. (2017). Emerging Trends and Application of the Internet of Things (1st ed.). New Jersey: IGI Global.

[2] Bor, M., Vidler, J., \& Roedig, U. (2016). LoRa for the Internet of Things, International Conference on Embedded Wireless Systems and Networks. Graz, Austria.

[3] Malaysia Meteorology Department. (2016). Annual Report 2016. Ministry of Science, Technology and Innovation.

[4] Adelantado, F., Vilajosana, X., Tuset-Peiro, P., Martinez, B., Melia-Segui, J., \& Watteyne, T. (2017). Understanding the limits of LoRaWAN. IEEE Communications Magazine, 55, 9, 34-40.

[5] A Technical Overview of LoRa and LoRaWAN. (2015). 1st ed. LoRa Alliance, pp.1-10.

[6] Cattani, M., Boano, C. A., \& Romer, K. (2017). An experimental evaluation of the reliability of LoRa long-range low-power wireless communication. Journal of Sensor and Actuator Networks, 6, 7, 1-19.

[7] Kontogiannis, S., Kokkonis, G., Ellinidou, S. \& Valsamidis, S. (2017). Proposed fuzzy-NN algorithm with LoRa communication protocol for clustered irrigation systems. Future Internet, 9, 78, 1-23.

[8] Hsieh, C. L., Ye, Z. W., Huang, C. K., Lee, Y. C., Sun, C. H., Wen, T. H., Juang, J. Y., \& Jiang, J. A. (2017). A vehicle monitoring system based on the LoRa technique. World Academy of Science, Engineering and Technology International Journal of Transport and Vehicle Engineering, 11, 5.

[9] LG01 LoRa Gateway User Manual. (2017). 1st ed. [ebook]

[10] Beijing: Dragino. Available at: http://www.dragino.com/ [Accessed 8 Apr. 2017]. 